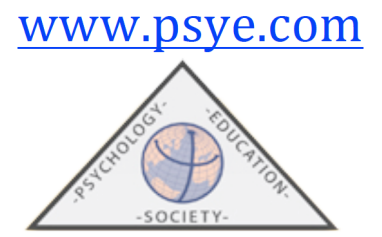

\title{
Desarrollo Profesional Docente: Perspectivas y Enfoques Internacionales
}

\author{
Alfredo BAUTISTA* \& Rosario ORTEGA-RUIZ**
}

(*) Nanyang Technological University, National Institute of Education (Singapur)

(**) Universidad de Córdoba, Facultad de Psicología (España)

\begin{abstract}
RESUMEN: En la actualidad, numerosas naciones en el mundo se encuentran embarcadas en profundas reformas de sus sistemas educativos. Existe un acuerdo generalizado entre políticos educativos, académicos y educadores en que una de las claves para el éxito de dichas reformas es fomentar el desarrollo profesional docente (DPD). Cada año, los gobiernos invierten cantidades astronómicas de dinero en el aprendizaje continuo de los profesores. Sin embargo, la literatura indica que buena parte del DPD ofrecido a los profesores es ineficaz, teniendo un efecto reducido o incluso nulo sobre sus prácticas y/o sobre el aprendizaje de los alumnos. Este monográfico describe las perspectivas y enfoques de DPD en cinco naciones altamente comprometidas con la investigación y/o la práctica en este campo. Conocer cómo el DPD se estructura en tales naciones puede ayudar a otras a diseñar oportunidades de aprendizaje más favorables para sus profesores. El artículo de Estados Unidos ofrece un marco general respecto a las características del DPD de alta calidad y ofrece ejemplos de recientes iniciativas eficaces. Los cuatro artículos siguientes describen los modelos de DPD en Australia, Hong Kong, Finlandia y Singapur, algunos de los países con sistemas educativos más exitosos. Dado que el aprendizaje continuo del profesorado se considera una prioridad, estas naciones han desarrollado sólidas infraestructuras de DPD para responder a las necesidades e intereses de los profesores. El monográfico concluye con una contribución de España, el país donde se edita la revista Psicología, Sociedad y Educación. La autora discute los cinco artículos anteriores y reflexiona sobre cómo las ideas presentadas podrían mejorar el DPD ofrecido actualmente a los profesores de otras naciones, en particular de España.
\end{abstract}

Palabras clave: desarrollo profesional, profesores en ejercicio, cambio educativo, educación comparativa

\section{Teacher Professional Development: International Perspectives and Approaches}

ABSTRACT: Nations around the world are currently embarked in deep reforms of their
education systems. There is widespread agreement among policymakers, scholars, and
educators that one of the keys for success during these reforms is promoting the
professional development (PD) of in-service teachers. Every year, governments invest 
astronomical amounts of money on teacher continuous learning. However, the literature shows that much of the PD offered to teachers is inefficient, having small or no effect on teaching practices and/or student learning. This monograph describes the perspectives and approaches to teacher PD of five nations heavily committed to research and/or practice in this field. Understanding how PD is structured in these nations may guide others in designing more favorable learning opportunities for their teachers. The article from United States provides a general framework regarding the features of high-quality PD and offers examples of recent effective initiatives. The four following articles describe the PD models of Australia, Hong Kong, Finland, and Singapore, among the highest-achievers in education presently. Because teacher continuous learning is a high priority in these nations, strong infrastructures for high-quality PD have been built to meet teachers' needs and interests. The monograph closes with a contribution from Spain, the country where the journal Psychology, Society and Education is edited. The author discusses the five prior articles and reflects on how the ideas presented could improve the PD currently offered to teachers in other nations, particularly Spain.

Keywords: professional development, in-service teachers, education change, comparative education

Correspondencia: Alfredo Bautista, Research Scientist \& Lecturer. Nanyang Technological University - National Institute of Education. Education \& Cognitive Development Lab. 1 Nanyang Walk. NIE5-B3-16. Singapur [637616]. Teléfono: (+65) 6219 6256. Fax: (+65) 6896 9845. E-mail: Alfredo.Bautista@nie.edu.sg

\section{Cómo citar este artículo}

Bautista, A., \& Ortega-Ruíz, R. (2015). Desarrollo professional docente: Perspectivas y enfoques internacionales (trad. al castellano de A. Bautista). Psicología, Sociedad y Educación, 7(3). 343-355 [V.O.: Teacher professional development: International perspectives and approaches. Psychology, Society and Education, 7(3). 240-251].

\section{Introducción}

Vivimos en un mundo cada vez más globalizado, donde los países están sujetos a intensos cambios de naturaleza económica, política y tecnológica. La educación está experimentando también rápidas transformaciones como resultado de los efectos de la globalización. Sin embargo, pese a su importancia para moldear nuestro futuro, la educación no siempre se analiza a la luz de los parámetros que han producido resultados de alta calidad en ciertos sistemas educativos. Las políticas locales continúan prevaleciendo sobre el conocimiento científico a la hora de determinar el rumbo de la mayoría de los países en materia de educación. Este monográfico parte de la idea de que hacer explícitos los parámetros que definen una educación de calidad es esencial en nuestro actual mundo globalizado. En particular, el presente monográfico se centra en formas efectivas de promover y facilitar el desarrollo profesional del profesorado en el siglo XXI.

El desarrollo profesional docente (DPD) implica numerosos procesos, acciones y mecanismos que están inevitablemente mediados por las características y 
condiciones particulares de cada contexto a nivel cultural, social, político y económico (Tan \& Dimmock, 2014). En el marco de una perspectiva teórica sistémica (situacional, contextual, ecológica), nuestro interés en este monográfico es presentar las perspectivas y enfoques de DPD adoptados en ciertas naciones seriamente comprometidas con la investigación y/o la práctica en este campo. Algunas de las preguntas que motivaron el presente monográfico fueron: ¿Qué modelos de DPD adoptan los países que han desarrollado las iniciativas de DPD más efectivas y/o innovadoras en los últimos años? ¿Cuáles son las semejanzas en el modo en que los profesores de dichos países participan en DPD? ¿Cuáles son las diferencias? ¿Con qué tipo de desafíos o problemas se encuentran estas naciones? ¿Cómo median los factores culturales, sociales, políticos y económicos en las prácticas de DPD existentes en el mundo? Consideramos que explorar estas cuestiones es una interesante tarea, con potencial para enriquecer nuestro conocimiento sobre los mecanismos y procesos que promueven el aprendizaje de los profesores en nuestro actual mundo globalizado.

\section{Los Profesores en Tiempos de Reformas Educativas}

Numerosas naciones en el mundo se encuentran actualmente embarcadas en profundas reformas de sus sistemas educativos. Uno de los cambios más sustanciales introducidos por las reformas tiene que ver con la dramática transformación en la naturaleza y tipos de resultados de aprendizaje esperados por parte de los estudiantes. Los países han establecido ambiciosas metas de aprendizaje, incluyendo tanto resultados académicos como no-académicos (Todd, 2010).

El propósito de la escuela hoy día no es simplemente transmitir contenidos disciplinares (matemáticas, ciencia, lengua, etc.) y preparar a los estudiantes para sus futuras carreras profesionales. El propósito es educar a los ciudadanos del siglo XXI: aprendices activos, auto-dirigidos, seguros de sí mismos y comprometidos, competentes a nivel cognitivo, emocional, social y tecnológico. También se considera importante preparar a los estudiantes para tomar decisiones responsables y equiparles con las llamadas competencias y habilidades del siglo XXI (p.ej., ser capaz de pensar crítica y creativamente, de comunicarse y colaborar con otros de forma efectiva, ser consciente de los problemas globales e inter-culturales) (Burnaford, Brown, Doherty, \& McLaughlin, 2007). Otro cambio importante introducido por las reformas en muchas naciones tiene que ver con la meta de promover la igualdad y la justicia social en las escuelas (p.ej., Apple, 2001). Se espera que las escuelas promuevan dichos valores de manera igualitaria y efectiva con todos los alumnos, incluso en las clases más diversas, contribuyendo de este modo a una sociedad mejor, más justa y libre (Kaur, 2012).

Cambios de esta magnitud necesariamente requieren de profundas transformaciones a nivel curricular e instruccional, en el qué y en el cómo los profesores enseñan a sus alumnos (Bautista, Tan, Ponnusamy, \& Yau, 2015). En efecto, los profesores son clave para el éxito de las reformas educativas, dado que ellos son los últimos responsables de poner en práctica las nuevas iniciativas dentro de las aulas (Guskey, 2002). En el siglo XXI, la expectativa es que los profesores 
cumplan numerosos roles dentro de las escuelas. Asegurar que los profesores están adecuadamente equipados con este sofisticado conjunto de competencias (p.ej., conocimientos y habilidades relacionadas con la práctica profesional, colaboración y liderazgo, integridad y compromiso con la educación y el cambio educativo, etc.) es por tanto esencial para garantizar el éxito de las reformas (Garet, Porter, Desimone, Birman, \& Kwang, 2001). Sin embargo, la investigación ha mostrado que muchos profesores necesitan importantes apoyos y orientación para ser capaces de enseñar de acuerdo a principios innovadores (Borko, 2004). De hecho, algunos investigadores han sugerido que muchas iniciativas de innovación educativa han fracasado en el pasado precisamente por no ofrecer a los profesores las oportunidades de aprendizaje apropiadas (Fullan \& Miles, 1992).

Existe un acuerdo generalizado entre políticos educativos, académicos y educadores en que promover el desarrollo profesional de los docentes (DPD) es una de las claves para el éxito de las reformas educativas (Desimone, Porter, Garet, Yoon, $\&$ Birman, 2002). En efecto, existe gran consenso en que la calidad de un sistema educativo no puede ser superior a la calidad de sus profesores (Barber \& Mourshed, 2007). Por esta razón, muchas naciones en el mundo están invirtiendo hoy día en el aprendizaje continuo de sus profesores como principal motor para mejorar tanto las competencias docentes como el rendimiento académico de los alumnos (DarlingHammond, Chung Wei, \& Andree, 2010). Como señala Knight (2002), ofrecer oportunidades de desarrollo profesional es esencial porque los programas iniciales de formación no pueden proporcionar a los docentes todas las competencias que se necesitan en el aula, especialmente las habilidades procedimentales ("saber cómo"), que se desarrollan principalmente en contextos prácticos. La expectativa es que el aprendizaje de los profesores se extenderá a lo largo de toda su carrera, lo cual les permitirá adaptarse a nuevas situaciones y responder a las demandas cambiantes de la sociedad en clase. Asimismo, ofrecer a los profesores oportunidades de DPD generalmente contribuye a mejorar su compromiso y satisfacción con el trabajo, reduciendo las tasas de abandono de la profesión docente (Dede, Ketelhut, Whitehouse, Breit, \& McCloskey, 2009).

\section{Definiendo el Desarrollo Profesional Docente (DPD)}

La literatura presenta numerosas conceptualizaciones con respecto al foco, el alcance y las metas del desarrollo profesional docente (DPD). Uno de los aspectos donde no existe consenso es la propia audiencia del DPD. La mayoría de los autores dentro de la comunidad académica anglosajona considera que el DPD sólo compete a los profesores en ejercicio, dado que únicamente éstos pueden ser considerados como profesionales (Little, 1993). En esta perspectiva, se entiende que el DPD es equivalente a la educación permanente (o continua) del profesorado. En contraste, otros autores consideran que los programas de formación inicial también forman parte de la trayectoria profesional docente, argumentando por tanto que los estudiantes de dichos programas deben también considerarse como la audiencia del DPD (Niemi, 2015). Este monográfico incluye trabajos basados en ambas perspectivas. 
Respecto al foco y metas principales, autores como Borko (2004) y Desimone et al. (2002) conceptualizan el DPD como un mecanismo esencial para la mejora del conocimiento y de las prácticas de enseñanza de los profesores. Para otros autores como Guskey (2002), cambiar las actitudes y las creencias de los docentes es otro de los objetivos prioritarios. Más recientemente, Kazemi y Hubbard (2008) y Opfer y Peder (2011) han enfatizado la necesidad de conceptualizaciones más complejas, argumentando que el DPD puede afectar numerosos aspectos de la vida profesional y personal de los docentes, incluyendo sus conocimientos, competencias y valores. En este monográfico adoptamos la conceptualización del DPD propuesta por Avalos (2011), dado que articula con elegancia diversos tópicos relevantes discutidos por los investigadores del campo en los últimos años. Como explica Avalos en su definición, entendemos que la meta última del DPD es beneficiar el aprendizaje y el rendimiento académico de los estudiantes.

"[...] el desarrollo profesional trata sobre el aprendizaje de los profesores, sobre cómo aprenden a aprender y a transformar su conocimiento en práctica para beneficiar el desarrollo de sus alumnos. El aprendizaje profesional docente es un proceso complejo que requiere la implicación cognitiva y emocional de los profesores tanto individualmente como colectivamente, la capacidad y disponibilidad para examinar dónde cada uno se encuentra en términos de convicciones y creencias, así como el análisis y la puesta en práctica de alternativas apropiadas para la mejora o el cambio." (Avalos, 2010; p.10, nuestra traducción).

El campo del DPD constituye un dominio de investigación de pleno derecho, con su propio corpus de teorías y modelos (Avalos, 2011). Durante sus tres décadas de vida, los investigadores han propuesto múltiples perspectivas teóricas sobre cómo los profesores aprenden y cambian. Estas teorías incluyen desde modelos unidireccionales (Guskey, 2002) y multidireccionales (Desimone, 2009), donde el aprendizaje del profesor es concebido como una consecuencia más bien directa de ciertas condiciones y procesos, hasta modelos basados en teorías del caos y la complejidad (Opfer \& Peder, 2011), donde el aprendizaje del profesor se conceptualiza como un resultado impredecible de procesos cíclicos y dinámicos.

La literatura presenta muchos tipos de estudios, de pequeña a gran escala, incluyendo diseños cuantitativos, cualitativos y mixtos. Las evaluaciones de programas son quizás el tipo de estudio más común. En dichas evaluaciones, los investigadores han analizado el impacto de programas de DPD específicos sobre los profesores (p.ej., hasta qué punto y cómo el DPD ha fomentado cambios en sus creencias, conocimientos o prácticas) y/o sobre los estudiantes (p.ej., hasta qué punto y cómo el DPD ha producido mejoras en su aprendizaje, o en sus puntuaciones en pruebas estandarizadas). Además, se han realizado estudios sobre las experiencias previas de los profesores en DPD, detallados análisis de prácticas de DPD exitosas, y más recientemente estudios experimentales (p.ej., ensayos aleatorizados por conglomerados) (para una síntesis de la literatura, véase Avalos, 2011). 


\section{Sobre la Efectividad del Desarrollo Profesional Docente (DPD)}

Los investigadores en DPD han acumulado una sólida base de conocimientos sobre qué funciona y qué no funciona en la práctica a la hora de promover el aprendizaje del profesor. La mayor parte de la investigación ha cosechado resultados decepcionantes respecto a la efectividad del DPD para ayudar a los docentes a mejorar sus conocimientos y sus prácticas (e.g., Garet et al., 2008, 2011; O'Dwyer et al., 2010; Powell et al., 2010). Cada año, los gobiernos invierten cantidades astronómicas de dinero en actividades tradicionales de DPD como seminarios, charlas, talleres y conferencias (Gersten, Dimino, Jayanthi, Kim, \& Santoro, 2010; Yoon, Duncan, Lee, Scarloss, \& Shapley, 2007), eventos breves y esporádicos donde los profesores tienden a ser receptores pasivos de información y donde no se les ofrecen oportunidades de colaborar con otros. Ball (1995) ha descrito este tipo de actividades como "shows de estilo" (style shows) y Darling-Hammond (2010) como "enfoque de disemina y reza" (spray and pray approach), dada la falta de estructuras para ofrecer a los profesores feedback y apoyo continuado. Investigadores como Borko (2004) consideran que este tipo de oportunidades de DPD son inadecuadas porque tienden a ser fragmentarias y superficiales intelectualmente, desconectadas de las prácticas de clase, y desconectadas de las necesidades e intereses de los profesores.

Estas actividades generalmente surgen de proveedores locales (quienes frecuentemente carecen de conocimientos relevantes sobre teoría y práctica del DPD) y tienen una duración y un alcance relativamente reducido. Asimismo, generalmente se llevan a cabo sin más evaluación formal que meras encuestas de satisfacción completadas por los profesores al final de cada evento (Darling-Hammond, 2010). Hoy sabemos que estas formas tradicionales de DPD tienen un potencial limitado o incluso nulo para mejorar las puntuaciones de "valor añadido" de los profesores, y por consiguiente ningún potencial para beneficiar a los estudiantes (Hill, Beiseigel, \& Jacob, 2013). Sin embargo, quizás por carecer de mejores alternativas o ideas (Little, 1993), numerosas escuelas y distritos escolares en el mundo continúan invirtiendo sus recursos en este tipo de eventos -ofreciendo talleres cortos, invitando a profesores universitarios a dar charlas especializadas, o enviando a sus profesores a costosas conferencias y convenciones una o dos veces por año. Los beneficios del dinero invertido en este sector continuará siendo escasos si las escuelas y los distritos escolares continúan financiando exclusivamente este tipo de actividades (Odden, Archibald, Fermanich, \& Alix Gallagher, 2002).

Como señala Guskey (2002), "para ser exitoso, el desarrollo profesional debe ser visto como un proceso, no como un evento" [to be successful, professional development must be seen as a process, not an event] (p.388) y necesita ofrecer a los profesores "ideas específicas, concretas y prácticas que se relacionen directamente con la realidad diaria de sus clases" [specific, concrete, and practical ideas that directly relate to the day-to-day operation of their classrooms] (p. 382). Estudios de encuesta llevados a cabo en los Estados Unidos con amplias muestras de profesores de distintas materias -especialmente matemáticas y ciencias- han identificado una serie de características relativas al contenido y al diseño del DPD que los docentes 
tienden a valorar positivamente. Los investigadores se han referido a ellas como "características del DPD de alta calidad" (p.ej., Bautista, Cañadas, Brizuela, \& Schliemann, 2015; Borko, 2004; Darling-Hammond \& McLaughlin, 2011; Desimone, 2009; Garet et al., 2001). De acuerdo a datos de auto-informe de los propios profesores, las actividades de DPD que presentan dichas características tienden a tener efectos positivos tanto en sus conocimientos como en sus prácticas instruccionales.

Respecto a las características relacionadas con el contenido, el DPD de alta calidad se centra en la materia/s específica que el profesor enseña en clase (p.ej., matemáticas, ciencias, historia), ofreciéndole así oportunidades para mejorar su propia comprensión de la materia en sí (contenido disciplinar), su comprensión de las estrategias pedagógicas necesarias para enseñar la materia a los estudiantes (conocimiento pedagógico), y su comprensión de cómo los estudiantes conceptualizan y aprenden la materia (conocimiento del pensamiento del estudiante) (Carpenter, Fennema, Peterson, Chiang, \& Loef, 1989; Penuel, Fishman, Yamaguchi, \& Gallagher, 2007). El DPD de alta calidad está "diseñado a la medida de los profesores" dependiendo de su conocimiento previo y de su nivel de pericia, es coherente con y responde a sus necesidades e intereses, y es consistente con los requerimientos curriculares de la escuela, del distrito y/o del estado. Finalmente, el DPD de alta calidad es voluntario y fomenta la autonomía y la elección en los profesores (Putnam \& Borko, 2000).

Respecto a las características de diseño (estructura y dinámicas de trabajo), el DPD de alta calidad ofrece a los profesores a) oportunidades de aprendizaje activo, incluyendo actividades para explorar, reflexionar y discutir; b) contextos para la participación colectiva y para compartir conocimientos con otros colegas; c) feedback constructivo y no-prescriptivo; y d) apoyo sostenido una vez finalizado el programa de DPD (Bautista et al., 2015; Desimone, 2009; Sherin \& Han, 2004). Los profesores necesitan extensos periodos de tiempo para reflexionar sobre las nuevas ideas que les son presentadas, para probarlas en sus clases y para discutir sobre ellas con sus colegas. De hecho, los investigadores han concluido que para fomentar verdaderamente el aprendizaje y el cambio del profesor, el DPD necesita ser intensivo y sostenido, en vez de corto y esporádico, implicando un número elevado de horas de contacto durante periodos de tiempo extensos. Se considera que las actividades de mayor duración ofrecen más oportunidades para analizar de forma integral el conocimiento disciplinar, las pedagogías y el pensamiento de los estudiantes (Garet et al., 2001). Del mismo modo, se considera que las actividades distribuidas en el tiempo, incluyendo más de 20 horas de contacto, son generalmente más efectivas (Desimone, 2009).

\section{Justificación y Estructura}

El título del presente monográfico publicado por Psicología, Sociedad y Educación (PSE) es "Desarrollo Professional Docente: Perspectivas y Enfoques Internacionales". El monográfico se fundamenta en una visión situada, contextual y ecológica del DPD. Como señalan Opfer y Peder (2011), la mayor parte de la 
literatura se ha centrado en analizar actividades o programas individuales, considerándolos de forma aislada de los complejos y dinámicos contextos donde los profesores participan. En otras palabras, los investigadores han tendido a centrarse fundamentalmente en los factores y contextos micro del DPD (p.ej., los efectos de actividades aisladas), ignorando las influencias de los factores y contextos meso (instituciones, sistema escolar) y macro (cultura, sociedad, política, economía).

Para llenar este vacío en la literatura, este monográfico presenta una serie de artículos en los que se describen las perspectivas y enfoques de DPD en cinco naciones seriamente comprometidas con la investigación y/o la práctica en este campo. En nuestra carta de invitación a los autores, les explicamos que estábamos interesados en aprender sobre las diferentes prácticas de DPD que hubiesen resultado particularmente exitosas, efectivas y/o beneficiosas para los profesores en sus respectivos países. Animamos a los autores a ofrecer descripciones generales de los modelos de DPD vigentes en sus respectivas naciones, clarificando que los artículos no debían basarse exclusivamente en sus trabajos o investigaciones previas. También les sugerimos una serie de preguntas guía para articular sus escritos, tales como: ¿Qué esta funcionando actualmente (o funcionando "mejor") en el DPD en su nación? ¿Cuáles son las bases teóricas de las perspectivas y enfoques adoptados hoy día? ¿Cuáles son las dificultades y problemas más importantes a los que se enfrenta el DPD en su nación? ¿Qué pueden otros países aprender del modo/s en que su país organiza actualmente el DPD? Se dio a los autores total libertad para abordar estas cuestiones u otras que considerasen más pertinentes o interesantes.

Invitamos a autores de cinco países que, de acuerdo a la literatura, han diseñado e implementado iniciativas de DPD especialmente efectivas y/o innovadoras en los últimos años. Una de las contribuciones proviene de los Estados Unidos, a buen seguro el país que actualmente lleva a cabo la investigación más puntera en el campo del DPD. También invitamos contribuciones de Australia, Hong Kong, Finlandia y Singapur, algunos de los países con los sistemas educativos más exitosos hoy día, con base en indicadores como los resultados de los estudiantes en comparaciones internacionales, las tasas de graduación, o el porcentaje de estudiantes que llevan a cabo estudios de postgrado (World Economic Forum's Global Competitiveness Report 2014-2015; véase Schwab, 2015). La literatura ha mostrado en estas naciones han desarrollado sólidos sistemas para promover el cambio docente, basándose en los principios del aprendizaje profesional efectivo descritos en la literatura educativa (Wei, Darling-Hammond, Andree, Richardson, \& Orphanos, 2009). El DPD es considerado una alta prioridad en estas naciones y los profesores son altamente respectados como profesionales (Darling-Hammond et al., 2010). Comprender más sobre cómo estas naciones están logrando buenos resultados puede informar las políticas y prácticas de DPD de otros países en el mundo. El monográfico concluye con una contribución de España, el país donde se edita la revista Psicología, Sociedad y Educación. La autora discute los cinco artículos anteriores y reflexiona sobre cómo las ideas presentadas podrían mejorar el DPD ofrecido a los profesores de otras naciones, en particular de España.

A continuación, procedemos a introducir los diferentes artículos incluidos en el (C) Psy, Soc, \& Educ, 2015. Vol. 7(3) 
monográfico, siguiendo el mismo orden con que son presentados.

\section{Estados Unidos de America}

Laura M. Desimone y Michael S. Garet son los autores de "Mejores Prácticas de Desarrollo Profesional Docente en Estados Unidos". Este artículo proporciona al monográfico un marco conceptual general sobre el DPD efectivo. Los autores comienzan describiendo su modelo original sobre las características críticas del "DPD de alta calidad" (Desimone, 2009), el cual sugiere que el DPD exitoso requiere la existencia de cinco rasgos clave: foco en el contenido, aprendizaje activo, coherencia, duración sostenida y participación colectiva. Los autores continúan discutiendo varios estudios realizados recientemente en USA, donde se han puesto a prueba estas cinco características. Con base en los resultados obtenidos, los autores proponen una versión mejorada de su modelo original. Desimone y Garet examinan también las recientes tendencias en la evolución del DPD en USA y finalizan discutiendo las dificultades que las escuelas y los distritos escolares encuentran a la hora de implementar DPD de alta calidad (Desimone \& Garet, 2015).

\section{Australia}

El título de la segunda contribución es "Una perspectiva australiana sobre el desarrollo profesional docente en tiempos supercomplejos". En este artículo, Lorraine M. Ling y Noella M. Mackenzie conceptualizan el DPD como un proceso que implica múltiples agentes y que recibe las influencias tanto de los gobiernos como de otros organismos externos. Las autoras describen los diversos tipos de iniciativas de desarrollo profesional ofrecidos actualmente a los profesores en Australia, ofreciendo ejemplos ilustrativos de diferentes materias y niveles educativos. Asimismo, las autoras reflexionan sobre el tipo de oportunidades de DPD que los profesores necesitan para enfrentarse a la "era de la supercomplejidad", caracterizada por la incertidumbre, la inseguridad y por presentar un futuro desconocido e incierto. Ling y Mackenzie presentan una interesante comparación entre las oportunidades de DPD existentes en Australia y las oportunidades que, desde su punto de vista, serían más deseables en los actuales tiempos supercomplejos (Ling \& Mackenzie, 2015).

\section{Finlandia}

Hannele Niemi es la autora de la tercera contribución, titulada "Desarrollo profesional docente en Finlandia: Hacia un enfoque más holístico". Este artículo define el DPD como un continuo que abarca toda la carrera profesional del profesor, incluyendo los programas de formación inicial. Niemi ofrece una completa revisión de las distintas perspectivas y enfoques de DPD en Finlandia. En dicho país se entiende que los profesores han de ser los principales promotores de su desarrollo personal y del desarrollo de las escuelas. Incluso durante la formación inicial, se ofrece a los profesores oportunidades para trabajar en contextos que fomentan su autonomía y agencia interna. Los profesores finlandeses adquieren sólidas competencias investigadoras, lo cual les prepara para diseñar proyectos de desarrollo e innovación escolar. En este artículo, Niemi describe cuatro casos que ilustran cómo 
apoyar el DPD de los profesores a través de la cooperación entre diferentes profesionales, cómo promover la innovación pedagógica a partir del diseño de investigaciones, cómo conectar la educación inicial y permanente del profesorado a través de la investigación, y cómo ofrecer apoyo a los profesores noveles mediante periodos de inducción (Niemi, 2015).

\section{Hong Kong}

El cuatro artículo, escrito por Bick Har Lam (2015), lleva por título "Desarrollo profesional docente en Hong Kong comparado con el mundo anglosajón: El rol de la filosofía de Confucio". Este artículo presenta una detallada revisión de las políticas y prácticas de DPD adoptadas en Hong Kong durante las cuatro últimas décadas, comparándolas con las políticas y prácticas adoptadas en varias naciones anglosajonas. El análisis documental se utiliza como principal metodología de investigación. Lam muestra que, al igual que en otros países, el DPD en Hong Kong ha pasado por fases claramente diferenciadas, comenzando por un enfoque puramente centrado en entrenar a los profesores mediante cursos de actualización pedagógica, hasta llegar un enfoque que concibe el DPD como aprendizaje continuo y permanente. Sin embargo, la autora argumenta que el hecho de que Hong Kong es una cultura de tradición confuciana ha introducido importantes diferencias en el modo en que los profesores participan en actividades de DPD. A partir de una discusión profunda de estas diferencias, Lam plantea conclusiones e implicaciones dirigidas a ayudar a otros países a mejorar sus políticas y prácticas de DPD.

\section{Singapur}

El título de la quinta contribución es "Desarrollo profesional docente en Singapur: Describiendo el panorama". Sus autores son Alfredo Bautista, Joanne Wong y Saravanan Gopinathan. Este artículo ofrece una descripción general de los recursos de DPD ofrecidos actualmente a los profesores de las escuelas primarias y secundarias gestionadas por el Ministerio de Educación de Singapur. Existen numerosos tipos de actividades donde estos profesores pueden participar durante sus 100 horas anuales de DPD voluntario, desde cursos-programas formales y estructurados hasta iniciativas más informales e innovadoras (investigación-acción, "lesson study"). La mayoría del DPD se centra en contenidos escolares específicos y proporciona a los profesores oportunidades para el aprendizaje colaborativo y para compartir conocimientos con sus colegas de forma colegial. Los autores argumentan que el conjunto de recursos ofrecido en Singapur, considerado de forma integral, presenta las características del DPD de alta calidad descritas en la literatura. Sin embargo, también sugieren que es necesaria más investigación que examine hasta qué punto este ambicioso modelo está contribuyendo a mejorar las prácticas de los profesores y el aprendizaje de los estudiantes (Bautista, Wong, \& Gopinathan, 2015).

\section{España}

Elena Martín es la autora del artículo final, titulado "Caminos que se consolidan en el desarrollo profesional docente. ¿Están presentes en España?”. Este 
artículo cumple dos objetivos. En primer lugar, discute los cinco artículos presentados en el monográfico, analizando las características comunes en las distintas perspectivas y enfoques de DPD considerados hoy día de mayor calidad. Martín argumenta que los principales ejes para promover el cambio del profesor se articulan entorno al desarrollo continuo durante la carrera docente, a la reflexión dentro de comunidades de práctica en las escuelas, y al valor de las voces de los estudiantes. Asimismo, la autora defiende que las políticas del DPD necesitan ser coherentes con políticas más globales dirigidas a mejorar la calidad de la educación. En segundo lugar, utilizando los ejes de cambio propuestos como marco de análisis, Martín discute la situación del DPD en España. Su análisis plantea un panorama más bien desolador, con importantes limitaciones respecto tanto a las actividades específicas que se ofrecen a los profesores como al modelo de DPD subyacente (Martín, 2015).

Invitamos a los lectores de Psicología, Sociedad y Educación a reflexionar sobre cómo las ideas presentadas en los diferentes artículos de este monográfico podrían contribuir a mejorar la teoría, la política y la práctica del DPD en sus propios países.

\section{Referencias}

Apple, M. W. (2001). Comparing neo-liberal projects and inequality in education. Comparative Education, 37(4), 409-423.

Avalos, B. (2011). Teacher professional development in teaching and teacher education over ten years. Teaching and Teacher Education, 27(1), 10-20.

Ball, D. L. (1995). Developing mathematics reform: What don't we know about teacher learning -- but would make good working hypotheses? Paper presented at the Conference on Teacher Enhancement in Mathematics K-6, Arlington, VA.

Barber, M., \& Mourshed, M. (2007). How the world's best-performing school systems come out on top. London: McKinsey and Company.

Bautista, A., Cañadas, M. C., Brizuela, M. B., \& Schliemann, A. D. (2015). Examining how teachers use graphs to teach mathematics in a professional development program. Journal of Education and Training Studies, 3(2), 91-106. http://redfame.com/journal/index.php/jets/article/view/676/624

Bautista, A., Tan, L. S., Ponnusamy, L. D., \& Yau, X. (2015). Curriculum integration in Arts Education: Connecting multiple Art forms through the notion of 'space'. Journal of Curriculum Studies. DOI: 10.1080/00220272.2015.1089940

Bautista, A., Wong, J., \& Gopinathan, S. (2015). Teacher professional development in Singapore: Depicting the landscape. Psychology, Society and Education, 7(3), 311326.

Borko, H. (2004). Professional development and teacher learning: Mapping the terrain. Educational Researcher, 33(8), 3-15.

Burnaford, G. E., Brown, S., Doherty, J., \& McLaughlin, H. J. (2007). Arts integration. Frameworks, research, and practice: A literature review. Washington, DC: Council of Chief State School Officers.

Carpenter, T. P., Fennema, E., Peterson, P. L., Chiang, C. P., \& Loef, M. (1989). Using knowledge of children's mathematics thinking in classroom teaching: An experimental study. American Educational Research Journal, 26, 499-531. 
Darling-Hammond, L. (2010). Teaching for deeper learning: Developing a thinking pedagogy In A. P. C. Avila, C. Hui, J. H. Lin, J. C. Peng Tam, \& J. C. Lim (Eds.), Rethinking Educational Paradigms: Moving from Good to Great. CJ Koh Professorial Lecture Series No. 5 (pp. 13-18). Singapore: Office of Education Research, National Institute of Education.

Darling-Hammond, L., Chung Wei, R., \& Andree, A. (2010). How high-achieving countries develop great teachers. Stanford Center for Opportunity Policy in Education Research Brief, 1-8.

Darling-Hammond, L., Chung Wei, R., Andree, A., Richardson, N., \& Orphanos, S. (2009). Professional learning in the learning profession: A status report on teacher development in the United States and abroad. Standford University, CA: National Staff Development Council.

Dede, C., Ketelhut, D. J., Whitehouse, P., Breit, L., \& McCloskey, E. M. (2009). A research agenda for online teacher professional development. Journal of Teacher Education, $60(1), 8-19$.

Desimone, L. M. (2009). Improving impact studies of teachers' professional development: Toward better conceptualizations and measures. Educational Researcher, 38(3), 181199.

Desimone, L. M., \& Garet, M. S. (2015). Best Practices in Teachers' Professional Development in the United States. Psychology, Society and Education, 7(3), 252263.

Desimone, L. M., Porter, A. C., Garet, M. S., Yoon, K. S., \& Birman, B. F. (2002). Effects of professional development on teachers' instruction: Results from a three-year longitudinal study. Educational Evaluation and Policy Analysis, 24(2), 81-112.

Fullan, M. G., \& Miles, M. B. (1992). Getting reform right: What works and what doesn't. Phi Delta Kappan, 73, 745-752.

Garet, M. S., Cronen, S., Eaton, M., Kurki, A., Ludwig, M., Jones, W., . . Sztejnberg, L. (2008). The impact of two professional development interventions on early reading instruction and achievement (NCEE 2008-4030). Washington, DC: National Center for Education Evaluation and Regional Assistance, Institute of Education Sciences, U.S. Department of Education.

Garet, M. S., Porter, A. C., Desimone, L., Birman, B. F., \& Kwang, S. Y. (2001). What makes professional development effective? Results from a national sample of teachers. American Educational Research Journal, 38, 915-945.

Garet, M. S., Wayne, A. J., Stancavage, F., Taylor, J., Eaton, M., Walters, K., \& Doolittle, F. (2011). Middle school mathematics professional development impact study: Findings after the second year of implementation. (NCEE 2011-4025). Washington, DC: National Center for Education Evaluation and Regional Assistance, Institute of Education Sciences, U.S. Department of Education.

Gersten, R., Dimino, J., Jayanthi, M., Kim, J. S., \& Santoro, L. E. (2010). Teacher study group: Impact of the professional development model on reading instruction and student outcomes in first grade classrooms. American Educational Research Journal, 47, 694-739.

Guskey, T. R. (2002). Professional development and teacher change. Teachers and Teaching: theory and practice, 8(3/4), 381-389.

Hill, H. C., Beisiegel, M., \& Jacob, R. (2013). Professional development research: Consensus, crossroads, and challenges. Educational Researcher, 42(9), 476-487. 
Kaur, B. (2012). Equity and social justice in teaching and teacher education. Teaching \& Teacher Education, 28, 485-492.

Kazemi, E., \& Hubbard, A. (2008). New directions for the design and study of professional development: Attending to the coevolution of teachers' participation across contexts. Journal of Teacher Education, 59(5), 428-441.

Knight, P. (2002). A systemic approach to professional development: learning as practice. Teaching and Teacher Education, 18(3), 229-241.

Lam, B. H. (2015). Teacher Professional Development in Hong Kong Compared to Anglosphere: the Role of Confucian Philosophy. Psychology, Society and Education, 7(3), 295-310.

Ling, L. M., \& Mackenzie, N. M. (2015). An Australian perspective on teacher professional development in supercomplex times. Psychology, Society and Education, 7(3), XXXX.

Little, J. W. (1993). Teachers' professional development in a climate of educational reform. Educational Evaluation and Policy Analysis, 15(2), 129-151.

Martín, E. (2015). Pathways that converge in teacher professional development: Are they present in Spain? Psychology, Society and Education, 7(3), 327-342.

Niemi, H. (2015). Teacher professional development in Finland: Towards a more holistic approach. Psychology, Society and Education, 7(3), 279-294.

O’Dwyer, L. M., Master, J., Dash, S., De Kramer, R. M., Humez, A., \& Russell, M. (2010). E-learning for educators: Effects of on-line professional development on teachers and their students: Findings from four randomized trials: Retrieved from http://www.bc.edu/research/intasc/PDF/EFE_Findings2010_Report.pdf.

Odden, A., Archibald, S., Fermanich, M., \& Alix Gallagher, H. (2002). A cost framework for professional development. Journal of Education Finance, 28(1), 51-74.

Opfer, V. D., \& Peder, D. (2011). Conceptualizing teacher professional learning. Review of Educational Research, 81(3), 376-407.

Penuel, W. R., Fishman, B. J., Yamaguchi, R., \& Gallagher, L. P. (2007). What makes professional development effective? Strategies that foster curriculum implementation. American Educational Research, 44, 921-958.

Powell, D. R., Diamond, K. E., Burchinal, M. R., \& Koehler, M. J. (2010). Effects of an early literacy professional development intervention on head start teachers and children. Journal of Educational Psychology, 102(2), 299-312. doi: $10.1037 / \mathrm{a} 0017763$

Putnam, R. T., \& Borko, H. (2000). What do new views of knowledge and thinking have to say about research on teacher learning? Educational Researcher, 29(1), 4-15.

Schwab, K. (2015). World Economic Forum's Global Competitiveness Report, 2014-2015. Retrived from: http://reports.weforum.org/global-competitiveness-report-20142015/. Switzerland: World Economic Forum.

Sherin, M. G., \& Han, S. Y. (2004). Teacher learning in the context of a video club. Teaching and Teacher Education, 20, 163-183.

Tan, C. Y., \& Dimmock, C. (2014). How a 'top-performing'Asian school system formulates and implements policy: the case of Singapore. Educational Management Administration \& Leadership, 1741143213510507.

Todd, R., J. (2010). Curriculum integration. Learning in a chanching world. Victoria, AU: McPerson's Printing Group. 
Wei, R. C., Darling-Hammond, L., Andree, A., Richardson, N., \& Orphanos, S. (2009). Professional learning in the learning profession: A status report on teacher development in the United States and abroad. Dallas, TX: National Staff Development Council. Downloaded from: http://edpolicy.stanford.edu.

Yoon, K. S., Duncan, T., Lee, S. W. Y., Scarloss, B., \& Shapley, K. L. (2007). Reviewing the evidence on how teacher professional development affects student achievement. Washington, DC: National Center for Educational Evaluation and Regional Assistance, Institute of Education Sciences, U.S. Department of Education. 